Article

\title{
Lateral Modes in Quantum Cascade Lasers
}

\author{
Gregory C. Dente ${ }^{1, *}$ and Michael Tilton ${ }^{2}$ \\ 1 GCD Associates, Albuquerque, NM 87110, USA \\ 2 The Boeing Company, Albuquerque, NM 87117, USA; Michael.L.Tilton@boeing.com \\ * Correspondence: GCDente@gmail.com; Tel.: +1-505-268-8337
}

Received: 18 February 2016; Accepted: 18 March 2016; Published: 22 March 2016

\begin{abstract}
We will examine the waveguide mode losses in ridge-guided quantum cascade lasers. Our analysis illustrates how the low-loss mode for broad-ridge quantum cascade lasers (QCLs) can be a higher-order lateral waveguide mode that maximizes the feedback from the sloped ridge-wall regions. The results are in excellent agreement with the near- and far-field data taken on broad-ridge-guided quantum cascade lasers processed with sloped ridge walls.
\end{abstract}

Keywords: quantum cascade lasers; ridge-guided; lateral modes

\section{Introduction}

Quantum cascade lasers (QCLs) are being used in applications requiring mid-infrared sources. For many of these applications, narrow-ridge devices can provide adequate power and beam quality. For applications requiring higher powers, broadening the ridge has led to higher-order lateral modes. These effects are displayed in recent experiments on broad-ridge quantum cascade lasers [1-3]. These near- and far-field studies of QCLs processed with sloping ridge walls have shown that as the ridge widths are increased, the lasing power is concentrated in just a few higher-order lateral waveguide modes. Consequently, the lasing lateral far-field forms a dual-lobed pattern that can remain relatively stable with nearly fixed divergence angles, independent of ridge width [2].

This paper will attempt to explain the dual-lobed far-field data taken on broad-ridge QCLs [2]. Many features could contribute to the dual-lobed far-fields. For instance, the sloping ridge walls vary with the processes used for the device fabrication. Factors such as dry- or wet-etch, wall slope and wall roughness, as well as the optical properties of the bounding regions, could all figure into a detailed analysis [4]. Here, we concentrate on one possible explanation. For our primary approximation, we analyze an ideal trapezoidal waveguide model, in which the top side has width $W-B$ and the bottom side has width $W+B$; the top and bottom are separated by a perpendicular distance, $D$. Figure 1 illustrates this ideal model for the QCL waveguide.

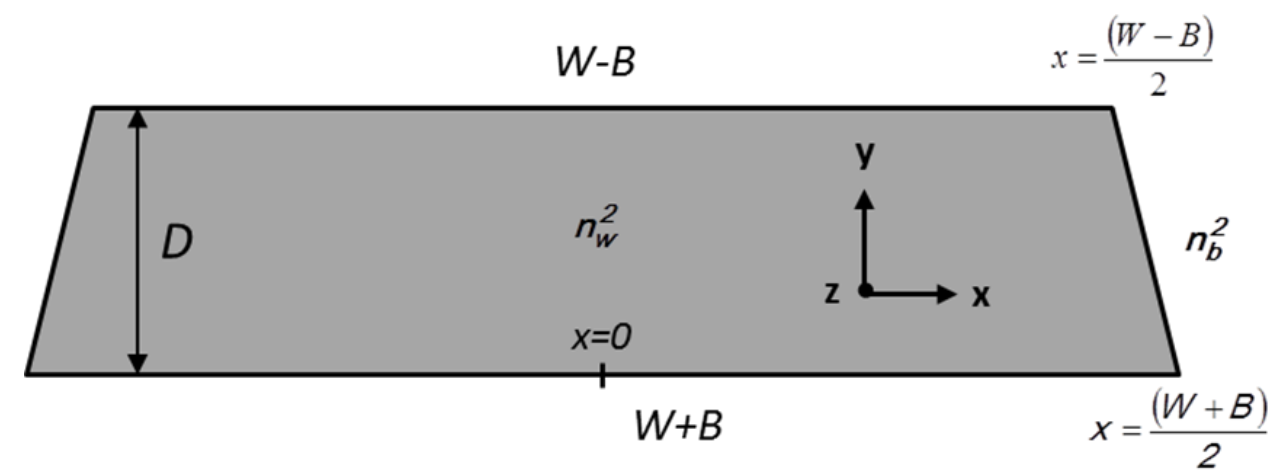

Figure 1. Trapezoidal waveguide model. 
Are we justified in replacing the complicated reality of the sloped ridge side walls with the ideal trapezoidal waveguide of Figure 1? Here, we will show that the lowest-loss cavity modes for the trapezoidal waveguide can explain the two-lobed far-field data [2]. In particular, the lateral extent of the sloped ridge-wall regions, $B$, proves to be the critical parameter. It determines the lowest-loss mode as a function of ridge width, and most importantly, demonstrates that the angular separation of the two lobes in the far-field is essentially independent of ridge width for the broad-ridge QCLs.

\section{Perturbation Approach to the Trapezoidal Waveguide Modes}

The ideal trapezoidal waveguide is difficult to analyze exactly. Therefore, we will use an approximate approach in which the trapezoidal waveguide is treated as a perturbation from the ideal rectangular waveguide shown in Figure 2.

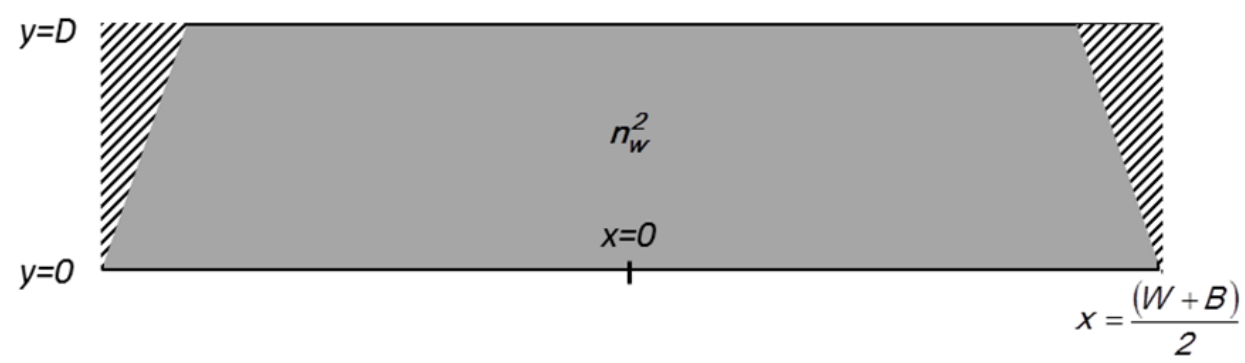

Figure 2. Rectangular waveguide model. Cross-hatched triangles carry perturbation $(2 \pi / \lambda)^{2} \cdot\left(n_{b}^{2}-n_{w}^{2}\right)$.

This rectangular waveguide propagates the electric field polarized in the epitaxial growth direction ( $y$-axis) between the back and front facets of the QCL. The ideal, unperturbed, rectangular waveguide modes are taken as either

$$
U_{N}(x, y)=\sin (\pi y / D) \cos (N \pi x /(W+B))
$$

in which $N$ is an odd integer for the symmetric lateral modes while

$$
U_{N}(x, y)=\sin (\pi y / D) \sin (N \pi x /(W+B))
$$

in which $N$ is an even integer for the anti-symmetric lateral modes [5]. For either case, the unperturbed $z$-component of the propagation vector is

$$
\beta_{0}(N)=\sqrt{\left(2 \pi n_{W} / \lambda\right)^{2}-(\pi / D)^{2}-(N \pi /(W+B))^{2}} .
$$

This form of ideal rectangular waveguide mode satisfies the wave equation in the guide region with complex index $n_{w}$. Also, the modes equal zero at the boundaries, $y=0, D$ and $x= \pm(W+B) / 2$. We could use a more exact form of mode in the y-direction, but as we will show, it has only a modest effect on the calculations that follow. Finally, we are assuming that these waveguide modes all operate coherently at a single wavelength, $\lambda$.

The propagation constant for the modes in a trapezoidal waveguide can be estimated from first-order perturbation theory as

$$
\beta^{2}(N) \approx \beta_{0}^{2}(N)+(2 \pi / \lambda)^{2}\left(n_{b}^{2}-n_{W^{2}}\right) \cdot\left[\iint_{\text {triangles }} U_{N}^{2} / \iint_{\text {rectangle }} U_{N}^{2}\right]
$$


in which $U_{N}(x, y)$ and $\beta_{0}(N)$ are the unperturbed rectangular waveguide results. We emphasize that the indices of refraction for both the waveguide and the barrier are complex numbers, so that the overall perturbation is complex.

The integral in the numerator is over the triangular cross-hatched regions shown in Figure 2, while the normalization in the denominator is over the entire rectangular waveguide cross-section. A final evaluation, after completing the lateral integration, gives the first-order perturbation theory estimate as

$$
\begin{aligned}
& \beta^{2}(N) \approx \beta_{0}^{2}(N)+(2 \pi / \lambda)^{2}\left(n_{b}^{2}-n_{W}^{2}\right) \\
& 4(B / D) /(D(W+B)) \cdot \int_{0}^{D} d y \sin ^{2}(\pi y / D)[y-\sin (f B y / D) /(f B / D)]
\end{aligned}
$$

in which $f=2 N \pi /(W+B)$. The parameters, $D, W, B$, as well as the complex indices of refraction for the waveguide and the barrier, are shown in the figures. Note that the perturbation changes both the real and imaginary parts of the propagation constant for the $\mathrm{N}$-order mode.

\section{Examples of QCL Cavity Mode Calculations}

The dual-lobed far-field data taken on broad-ridge QCLs is remarkable [2]. All the devices radiated 4.45 micron radiation into a pair of lobes at $\pm 38^{\circ}$ as shown in Figure 3 . Here, $W$ defines the device stripe width and $\mathrm{n}$ indicates the mode number.

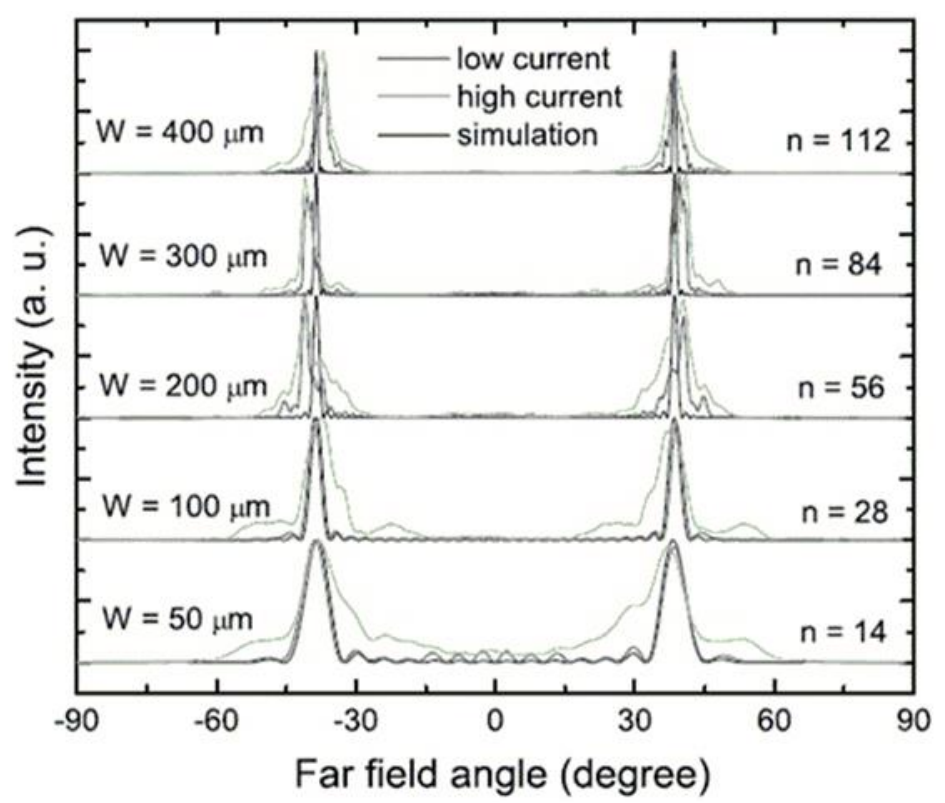

Figure 3. Far fields (Reference [2]) of broad area QCLs measured at both low $\left(1.1 \times I_{t h}\right)$ and high $\left(2 \times I_{t h}\right)$ current in pulsed mode operation at room temperature.

Additionally, while the individual lobes were not diffraction-limited, it is obvious that only a small set of higher-order lateral modes were operating. This behavior was observed, independent of ridge-width, from 50 micron wide devices out to 400 microns. Here, we will try to explain this behavior using the first-order perturbation result in Equation (3).

First, from Equation (3), we observe that a stationary value of the perturbation integral occurs when $f B / D=2 \pi / D$; at this value of $f=2 N \pi /(W+B)$, the second term in the integrand integrates to zero. This corresponds to $N \approx(W+B) / B$, in which $N$, the lateral mode order, must be an integer. Therefore, the perturbation solution suggests that the lowest-loss trapezoidal waveguide mode is 
of relatively high-order when $W>B$. We can insert this mode-order value, $N \approx(W+B) / B$ into the unperturbed mode expression as

$$
U_{N}(x, y)=\sin (\pi y / D) \sin (N \pi x /(W+B)) \approx \sin (\pi y / D) \sin (\pi x / B)
$$

so that the lowest-loss trapezoidal waveguide modes propagate to a dual-lobed far-field at lateral angles given by $\sin \theta= \pm \lambda /(2 B)$; for $\lambda=4.45 \mu \mathrm{m}$ and $\theta= \pm 38.5^{\circ}$, we calculate the lateral width of the sloping ridge side-walls as $B=3.57 \mu \mathrm{m}$. This value seems reasonable for the processing used on the Reference [2] devices. Furthermore, as long as we select the lowest-loss trapezoidal waveguide mode, these dual-lobed far-field angles and the near-field intensity periodicity do not depend on ridge width. Finally, we have defined the mode order at the base of the trapezoid. If we shift our reference to the mode order at the center layer, $y=D / 2$, then $N=W / B$. For the Reference [2] devices, this gives perfect agreement with the data in Figure 3, with mode orders $N=14,28,56,84$ and 112 at $B=3.57 \mu \mathrm{m}$.

\section{Conclusions}

The perturbation theory result, Equation (3), gives surprisingly good agreement with the data on broad-ridge QCLs. Normally, first-order perturbation theory is most reliable when we are evaluating small perturbations. The change from a rectangular waveguide to a trapezoidal waveguide does not, necessarily, seem that small. But perhaps for the broad-ridge QCLs, the effects at the sloping ridge walls can be viewed as only a small part of the overall waveguide, so that the perturbation is of order $B / W$; this appears to be the case here. An additional element of support can be developed from the two-slab approximation to the trapezoidal waveguide; this is illustrated in Figure 4.

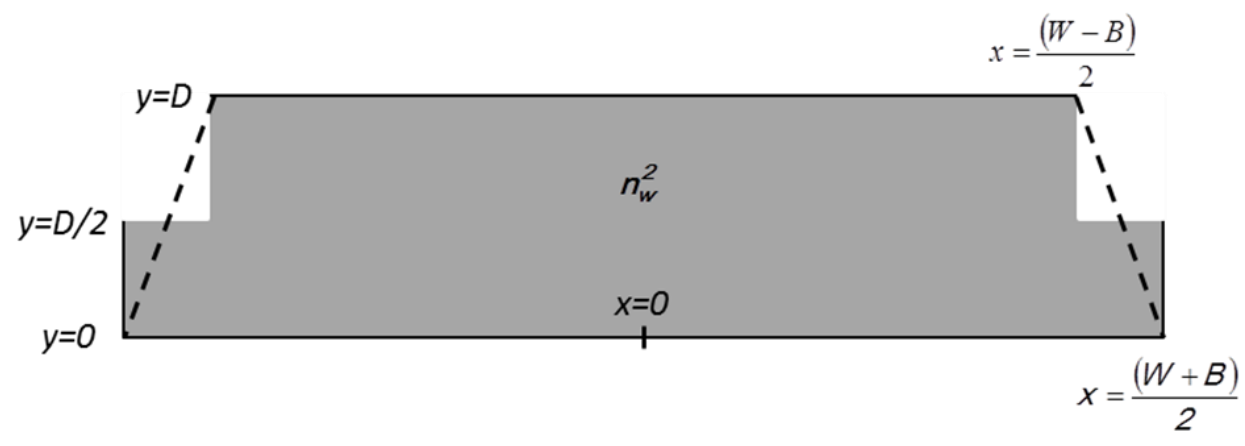

Figure 4. Two slab approximation.

We approximate the trapezoid with a slab of width $(W-B)$ centered over a slab of width $(W+B)$. We then take the lateral mode trial functions as $\sin (P x)$ or $\cos (P x)$ and force zero at the lateral boundaries, $x= \pm(W+B) / 2$ and $x= \pm(W-B) / 2$. The lateral boundary conditions for the two slab widths are satisfied when $P=N \pi /(W+B)$ with $N \approx(W+B) / B$; this is the same lateral mode order that resulted from the perturbation theory results in Equation (3).

It appears that feedback from the sloping ridge walls is enhanced for higher-order lateral modes. This result was suggested from both first-order perturbation theory as well as from the two-slab approximation. A lowest-loss lateral QCL mode of order $N \approx(W+B) / B$ is in excellent agreement with the near- and far-field data on broad-ridge QCLs [2]. Most importantly, the resulting dual-lobed far-field angles and the near-field intensity periodicity do not depend on ridge width.

Author Contributions: G. Dente conceived the analysis and wrote the manuscript. M. Tilton provided the comparison of theory and experiment as well as manuscript preparation support.

Conflicts of Interest: The authors declare no conflict of interest. 


\section{References}

1. Razeghi, M.; Bandyopadhyay, N.; Bai, Y.; Lu, Q.; Slivken, S. Recent Advances in Mid Infrared (3-5 $\mu \mathrm{m})$ Quantum Cascade Lasers. Opt. Mat. Exp. 2013, 3, 1873-1884. [CrossRef]

2. Bai, Y.; Slivken, S.; Darvish, S.R.; Haddadi, A.; Gokden, B.; Razeghi, M. High Power Broad Area Quantum Cascade Lasers. Appl. Phys. Lett. 2009, 95, 221104. [CrossRef]

3. Yu, N.; Diehl, L.; Cubukcu, E.; Pflügl, C.; Bour, D.; Corzine, S.; Zhu, J.; Höfler, G.; Crozier, K.B.; Capasso, F. Near-field Imaging of Quantum Cascade Laser Transverse Modes. Opt. Express 2007, 15, 13227-13235. [CrossRef] [PubMed]

4. Toor, F.; Sivco, D.L.; Liu, H.E.; Gmachl, C.F. Effect of Waveguide Sidewall Roughness on the Threshold Current Density and Slope Efficiency of Quantum Cascade Lasers. Appl. Phys. Lett. 2008, 93, 031104. [CrossRef]

5. Marcuse, D. Theory of Dielectric Optical Waveguides; Academic Press: New York, NY, USA, 1991.

(C) 2016 by the authors; licensee MDPI, Basel, Switzerland. This article is an open access article distributed under the terms and conditions of the Creative Commons by Attribution (CC-BY) license (http://creativecommons.org/licenses/by/4.0/). 